\title{
Análise da eficácia do óleo de hortelã pimenta (Mentha piperita L.) na síndrome do intestino irritável: revisão baseada em evidência
}

\author{
Analysis of the efficacy of the peppermint oil (Mentha piperita L.) on irritable bowel syndrome: \\ an evidence-based review \\ Análisis de la eficacia del aceite de menta (Mentha piperita L.) sobre el síndrome del intestino \\ irritable: revisión basada en evidencia
}

Joana Costa Gomes. Unidade de Saúde Familiar Lagoa - ULS Matosinhos. joanacostabgomes@hotmail.com (Autora correspondente)

Filipa Silva. Unidade de Saúde Familiar Dunas - ULS Matosinhos. fi_msilva@hotmail.com

\section{Resumo}

Objetivo: Avaliar a eficácia do óleo de hortelã-pimenta (Mentha piperita L) no tratamento de indivíduos com síndrome do intestino irritável (SII), analisando a melhora dos sintomas e da qualidade de vida. Métodos: Foram realizadas pesquisas nas bases de dados Trip database, National Guideline Clearinghouse, Guidelines finder, Cochrane Library, Dare, Bandolier e Medline utilizando os termos MeSH Irritable bowel syndrome e peppermint oil. Foram incluídas normas de orientação clínica (NOC), revisões sistemáticas e metanálises e ensaios clínicos aleatorizados e controlados (ECAC) que avaliassem a eficácia do óleo de hortelã-pimenta (OHP) no alívio sintomático ou melhoria da qualidade de vida de indivíduos com SII, quando comparado com placebo ou outra terapêutica validada. Resultados e Discussão: Foram selecionados 8 artigos (5 NOC, 3 revisões sistemáticas/ metanálises). A evidência sugere que 0 OHP é eficaz no alívio sintomático global da SII, especialmente quando existe dor abdominal, verificando-se a sua superioridade em relação ao placebo na maioria dos estudos avaliados - com odds ratio favorecendo o óleo de hortelã-pimenta atingindo 2,7 (IC de $95 \%$ de 1,6 a 4,8) e NNT de 3. Existe também evidência de melhoria da qualidade de vida com 0 OHP, em comparação com o placebo $(p<0,001)$. Conclusão: A evidência demonstra eficácia do OHP na SII, especialmente quando existe um componente de dor abdominal, quando comparado com o placebo (força de recomendação B). São necessários mais estudos com qualidade metodológica que avaliem eficácia e segurança a longo prazo do OHP no alívio sintomático da Sll.

\section{Abstract}

Objective: To evaluate the efficacy of peppermint oil (Mentha piperita L.) on individuals diagnosed with irritable bowel syndrome (IBS) regarding symptom improvement and quality of life enhancement. Methods: Literature search was conducted according to evidence based on methodology review at Trip database, National Guideline Clearinghouse, Guidelines finder, Cochrane Library, Dare, Bandolier and Medline, as well as using the MeSH index terms "Irritable bowel syndrome" and "peppermint oil". We selected practice guidelines, systematic reviews, meta-analysis and randomized controlled trials that evaluated peppermint oil (PO) efficacy in improving symptoms and/or quality of life when compared to placebo or other approved therapy for IBS. Results and Discussion: A total of eight articles were selected including five practice guidelines and three systematic reviews/meta-analysis. Existing evidence suggests that there are enough data to support the use of peppermint oil in IBS for overall symptomatic relief, especially when abdominal pain is the dominant symptom, when compared to placebo (with an odds ratio of 2.7; Cl 95\%, 1.6 to 4.8 and NNT of 3). There is also evidence regarding overall quality of life improvement when using PO compared to placebo $(\mathrm{p}<0.001)$. Conclusion: So far, evidence suggests that PO should be considered for IBS patients, especially in the presence of abdominal pain (Strength of recommendation B). However, more high methodological quality studies that evaluate long-term efficacy and security of PO are needed.

\section{Resumen}

Objetivo: Evaluar la eficacia del aceite de menta (Mentha piperita L.) en el tratamiento de pacientes con síndrome del intestino irritable (SII) y analizar el alivio de sus síntomas y la mejora en su calidad de vida. Métodos: Se realizaron búsquedas en las bases de datos Trip database, National Guideline Clearinghouse, Guidelines finder, Cochrane Library, Dare, Bandolier y Medline usando los términos MeSH "Irritable bowel syndrome" y "peppermint oil". Se incluyeron las normas de orientación clínica (NOC), revisiones sistemáticas y meta-análisis y ensayos clínicos controlados aleatorizados (ECAC) para evaluar la eficacia del aceite de menta (AM) en el alivio sintomático y mejora de la calidad de vida de las personas con SIl en comparación con el placebo u otra terapia validada. Resultados y Discusión: Se seleccionaron ocho estudios (cinco NOC y tres revisiones sistemáticas/meta-análisis). La evidencia sugiere que el AM es eficaz en el alivio de los síntomas generales del SII, en particular si había dolor abdominal, verificandose su superioridad sobre el placebo en la mayoría de los estudios evaluados (odds ratio de 2,7; IC 95\% de 1,6 a 4,8 y NNT de 3). Hubo también evidencia de mejora en la calidad de vida en los pacientes tratados con AM, en comparación con el placebo $(p<0,001)$. Conclusión: Las pruebas demuestran la eficacia del AM en el SII, especialmente en presencia de dolor abdominal, en comparación con el placebo (fuerza de recomendación B). Se necesitan más estudios con buena calidad metodológica que evalúen la eficacia y la seguridad a largo plazo del AM en el alivio de los síntomas del SII.

Como citar: Gomes JC, Silva F. Análise da eficácia do óleo de hortelã-pimenta na síndrome do intestino irritável: revisão baseada em evidência. Rev Bras Med Fam Comunidade. 2013;8(27):121-6. Disponível em: http://dx.doi.org/10.5712/rbmfc8(27)702

\section{Palavras-chave:} Síndrome do Intestino Irritável Óleo de Hortelã-pimenta Fitoterapia Preparações de Plantas

\section{Keywords:}

Irritable Bowel Syndrome Peppermint Oil Phytotherapy Plant Preparations

\section{Palabras clave:}

Síndrome del Colon Irritable Aceite de Menta Fitoterapia Preparados de Plantas 


\section{Introdução}

A síndrome do intestino irritável (SII) é uma patologia gastrointestinal funcional caracterizada por dor ou desconforto abdominal associada a alteraçóes do trânsito intestinal ${ }^{1,2}$. É uma patologia muito frequente, estimando-se que possa atingir até $20 \%$ de prevalência em alguns grupos populacionais ${ }^{3,4}$. Essa patologia crônica, frequentemente subdiagnosticada, com sintomatologia recorrente, não confere risco aumentado de mortalidade, embora se associe a morbilidade importante, diminuição da qualidade de vida, bem como custos diretos e indiretos consideráveis, levando o indivíduo a procurar cuidados médicos, frequentemente o seu médico de família, o que pode prejudicar a relação médico-paciente ${ }^{4-7}$.

Para diagnóstico dessa síndrome utilizam-se os critérios de Roma, atualmente na terceira revisão (Roma III), que estabelecem a necessidade da presença de dor abdominal ou desconforto (pelo menos três dias por mês nos últimos três meses), associada a duas das seguintes caraterísticas: alívio com a evacuação, alteraçáo da frequência das evacuaçóes e/ou alteração da forma das fezes ${ }^{8,9}$.

Essa síndrome tem abordagens terapêuticas diversas, dada a sua etiologia multifatorial, com envolvimento de múltiplos sistemas, incluindo-se fatores psicossociais, e seu controle sintomático é difícil com as terapêuticas convencionais ${ }^{10}$. Assim, têm surgido novas abordagens terapêuticas, tais como a fitoterapia, na qual se inclui o óleo de hortelã-pimenta (OHP). Esse fármaco deve ser administrado 3 vezes ao dia, na forma de cápsulas gastrorresistentes, cerca de 30 minutos antes das refeiçốes ${ }^{11}$.

O objetivo deste trabalho é verificar, com base na evidência científica, a eficácia do OHP na melhoria sintomática e da qualidade de vida de indivíduos com SII, de forma a garantir melhores cuidados a pacientes com essa doença.

\section{Métodos}

Foi realizada uma pesquisa bibliográfica nas bases de dados Trip database, National Guideline Clearinghouse, Guidelines finder, Cochrane Library, Dare, Bandolier e Medline, utilizando os termos MeSH Irritable bowel syndrome e peppermint oil. A pesquisa foi limitada às línguas portuguesa, inglesa e espanhola e a pesquisa de artigos originais foi limitada ao período compreendido entre janeiro de 2000 e janeiro de 2013.

Foram consideradas elegíveis normas de orientação clínica de sociedades médicas (Guidelines, Diretrizes Clínicas), revisōes sistemáticas/metanálises e ensaios clínicos aleatorizados e controlados (ECAC), utilizando-se uma metodologia que combinou revisão sistemática com revisão de revisões sistemáticas. Foram utilizados artigos que avaliassem a eficácia do OHP na SII, centrando a atenção na pergunta de pesquisa - "Será o OHP eficaz no alívio de pacientes com SII?" Os desfechos principais avaliados foram melhoria sintomática e/ou melhoria da qualidade de vida do doente em comparação com o placebo ou com outra terapêutica utilizada no tratamento da SII; secundariamente foram avaliadas a segurança e os efeitos colaterais desse fármaco. Foram excluídas revisôes clássicas, artigos de opinião, cartas ao editor, estudos observacionais e estudos repetidos/incluídos nas revisôes sistemáticas ou metanálises. Fez-se ainda uma revisão da bibliografia das revisões clássicas encontradas, de forma a verificar a existência de outros estudos que cumprissem os critérios de inclusão (Figura 1).

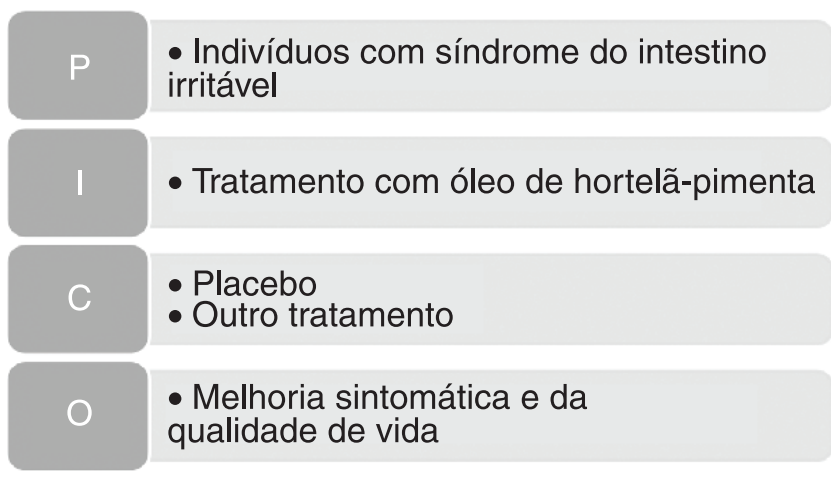

P, população; I, intervenção; C, controle;

O, outcome/desfecho

Figura 1. Revisão baseada em evidência. P, população; I, intervenção; C, controle; 0, outcome/desfecho 
A pesquisa foi feita pelas duas investigadoras de forma independente e posteriormente comparadas as seleçôes e discutidos os casos discordantes. Os artigos foram excluídos com base no título/abstract, se fosse claro que não se adequavam ao objetivo. Sempre que considerado necessário, foi obtido o texto integral e feita a leitura completa do artigo. Os artigos selecionados para avaliação foram lidos integralmente por ambas as investigadoras, que sistematizaram as informações em quadros estandardizados. Posteriormente foram discutidos os conteúdos desses quadros para a atribuição de níveis de evidência aos estudos incluídos, bem como para produzir conclusóes sumárias e respectivos graus de recomendaçáo. A qualidade dos estudos incluídos foi avaliada com base na taxonomia Strength of Recommendation Taxonomy (SORT) da American Academy of Family Physicians. Essa escala avalia parâmetros tais como o tipo de estudo, a qualidade da amostra, a coerência dos resultados, evidências centradas no paciente versus na doença, permitindo a atribuiçáo de níveis de evidência e forças de recomendação finais ${ }^{12}$.

\section{Resultados}

Da pesquisa bibliográfica inicial resultaram 104 artigos, dos quais foram incluídos 8 ( 5 normas de orientação clínica e 3 revisões sistemáticas) (Figura 2).

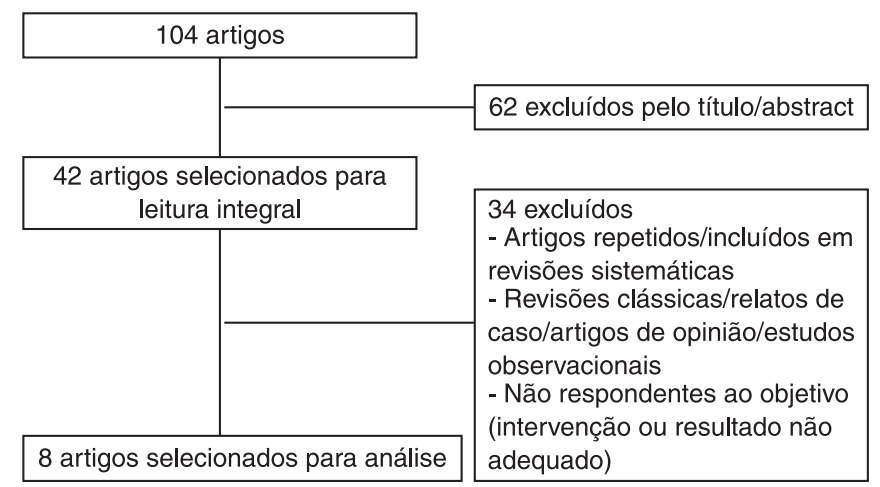

Figura 2. Organograma de seleção dos artigos para análise.

Quatro das NOC encontradas, todas com força de recomendação B, referiam-se à utilização do OHP na SII (tanto nos casos com predomínio de diarreia como naqueles com predomínio de obstipação) como terapêutica inicial associada a medidas dietéticas adequadas ${ }^{13}$, já que o OHP mostrou melhoria global da sintomatologia da SII ${ }^{14}$. Kligler e Chaudhary ${ }^{15}$ referem ainda que embora o OHP pareça ser uma alternativa segura existem poucos dados em relação à sua eficácia e segurança a longo prazo ${ }^{16}$. Uma das NOC refere que os ensaios clínicos que avaliaram a eficácia do OHP são demasiado pequenos para gerarem evidência convincente, uma vez que os seus resultados são contraditórios. De acordo com esta NOC, o seu uso só deveria ser recomendado após publicação de estudos com maior tamanho amostral (Quadro 1) ${ }^{17}$.

Quadro 1. Resumo das NOCs analisadas e respectivas forças de recomendação.

\begin{tabular}{|c|c|c|}
\hline Autor/ano/entidade & Conclusões & Grau de recomendação \\
\hline $\begin{array}{l}\text { Jones, } 2000 \\
\text { British Society of Gastroenterology }\end{array}$ & $\begin{array}{l}\text { Os ensaios clínicos que avaliaram a eficácia do OHP são demasiado pequenos para gerarem } \\
\text { evidência convincente e os seus resultados são contraditórios. } 0 \text { seu uso só deve ser recomendado } \\
\text { após publicação de estudos com maior tamanho amostral. }\end{array}$ & $B$ \\
\hline $\begin{array}{l}\text { Hadley, } 2005 \\
\text { American Academy of Family Physicians }\end{array}$ & $\begin{array}{l}\text { [...] OHP e ingestão adequada de líquidos é recomendada como terapêutica inicial em indivíduos } \\
\text { com SIl associado a predomínio de obstipação. } \\
\text { [...] OHP e manipulação da dieta são recomendados como terapêutica inicial em indivíduos com SII } \\
\text { com predomínio de diarreia. }\end{array}$ & B \\
\hline $\begin{array}{l}\text { Kligler, } 2007 \\
\text { American Academy of Family Physicians }\end{array}$ & $\begin{array}{l}\text { OHP parece ser uma alternativa segura na redução de sintomas de SII, embora a evidência seja } \\
\text { pouco clara. }\end{array}$ & B \\
\hline $\begin{array}{l}\text { Quigley, } 2009 \\
\text { World Gastroenterology Organisation }\end{array}$ & $\begin{array}{l}\text { OHP pode promover alívio da dor abdominal/desconforto, a curto prazo. Não há evidência da } \\
\text { eficácia a longo prazo e a evidência relativa a segurança e tolerabilidade é limitada. }\end{array}$ & B \\
\hline
\end{tabular}

OHP, óleo de hortelã-pimenta; SII, Síndrome do Intestino Irritável. 
Heizer $^{18}$ realizou em 2009 uma revisão sistemática que englobou duas revisões sistemáticas anteriores e um ECAC incluindo globalmente 20 estudos - nivel de evidência 2. Dos estudos incluídos, 13 mostraram superioridade do OHP em relaçáo ao placebo e três estudos revelaram que os efeitos do OHP foram semelhantes ao dos anticolinérgicos. O ECAC de Cappello et al. (2007), incluído nessa revisão, mostrou melhoria sintomática em $75 \%$ dos indivíduos tratados com OHP vs. 38\% dos indivíduos aos quais foi administrado placebo $(\mathrm{p}<0,05)^{18}$ (Quadro 2).

Quadro 2. Resumo das principais caraterísticas, resultados e conclusões das revisões sistemáticas/metanálises e ECAC analisados e atribuição de níveis de evidência.

\begin{tabular}{|c|c|c|c|c|c|}
\hline $\begin{array}{l}\text { Autor } \\
\text { (ano) }\end{array}$ & $\begin{array}{c}\text { Tipo de estudo/ } \\
\text { População }\end{array}$ & Intervenção & Resultados & Conclusões/Comentários & $\begin{array}{l}\text { Nível de } \\
\text { evidência }\end{array}$ \\
\hline $\begin{array}{l}\text { Heizer } \\
\text { (2009) }\end{array}$ & $\begin{array}{l}\text { Revisão sistemática } \\
\text { Incluiu: } \\
2 \text { revisões } \\
\text { sistemáticas e } 1 \text { ECAC }\end{array}$ & $\begin{array}{l}\text { OHP vs. placebo } \\
\text { OHP vs. outros } \\
\text { fármacos SII }\end{array}$ & $\begin{array}{l}8 \text { dos ECAC - superioridade da OHP em relação ao } \\
\text { placebo } \\
\text { Em } 3 \text { estudos - OHP semelhante aos anticolinérgicos } \\
\text { Melhoria sintomática } 75 \% \text { vs. } 38 \%(p<0,05)\end{array}$ & $\begin{array}{l}\text { OHP é o fármaco de primeira linha na SII } \\
\text { moderada pois a sua ingesta diária é eficaz no } \\
\text { controle sintomático da SII. }\end{array}$ & 2 \\
\hline $\begin{array}{l}\text { Shen } \\
\text { (2009) }\end{array}$ & $\begin{array}{l}\text { Revisão sistemática } \\
\text { Incluiu: } \\
2 \text { metanálises e } 1 \text { EC } \\
(n=533)\end{array}$ & $\begin{array}{l}\text { OHP vs. placebo } \\
4 \text { cápsulas/dia } \\
4 \text { semanas }\end{array}$ & $\begin{array}{l}\text { Efeito positivo global da OHP }(p<0,001) \\
\text { Melhoria sintomática com OHP } \\
(\mathrm{OR} 2,7, \text { IC } 95 \% 1,6-4,8)\end{array}$ & $\begin{array}{l}\text { Existe evidência suficiente para recomendar o uso } \\
\text { de OHP no tratamento da SII. } \\
\text { Heterogeneidade dos critérios de diagnóstico e } \\
\text { escalas de sintomas. } \\
\text { Generalização difícil (critérios de inclusão). }\end{array}$ & 2 \\
\hline $\begin{array}{l}\text { Ford } \\
\text { (2012) }\end{array}$ & $\begin{array}{l}\text { Revisão sistemática } \\
1 \text { revisão sistemática } \\
\text { de } 4 \text { ECAC }(n=392) \\
1 \text { revisão sistemática } \\
\text { de } 3 \text { ECAC }(n=326) \\
1 \text { ECAC }(n=90)\end{array}$ & OHP vs. placebo & $\begin{array}{l}\text { Melhoria sintomática com OHP } \\
\text { Melhoria sintomática } \\
\text { RR }=0,43 \text { (IC 95\% 0,32-0,59) } \\
\text { NNT = } 3 \\
\text { Melhoria da dor abdominal }(\mathrm{n}=101) \\
\text { RR }=2,15 \text { (IC 95\% 1,54-3,00) } \\
\text { Melhora sintomática global }(\mathrm{n}=225) \\
\text { RR = 2,25 (IC 95\% 1,70-2,98) } \\
\text { Proporção de indivíduos sem desconforto ou dor } \\
\text { abdominal } \\
42 \% \text { OHP vs. } 22 \% \text { placebo }(\mathrm{p}<0,001)\end{array}$ & $\begin{array}{l}\text { Os resultados dos estudos incluídos favorecem o } \\
\text { OHP quando comparado com o placebo. } \\
\text { Apenas dois dos estudos foram realizados no } \\
\text { contexto da atenção primária. } \\
\text { Alguns incluíram estudos com duração } \\
\text { insuficiente, sendo difícil avaliar a eficácia a } \\
\text { longo prazo. }\end{array}$ & 2 \\
\hline
\end{tabular}

ECAC: Ensaio Clínico Aleatorizado e Controlado; OHP: óleo de hortelã-pimenta.; SII: Síndrome do Intestino Irritável; EC: ensaio clínico; OR: odds ratio; IC: intervalo de confiança; RR: risco relativo.

A revisão sistemática de Shen ${ }^{19}$ incluiu duas metanálises: Pittler (1998) e Spanier (2003) e um ensaio clínico (EC), num total de 10 estudos $(\mathrm{n}=533)$. Os seus resultados revelaram um efeito positivo global do OHP ( $<<0,001)$ e melhoria sintomática com OHP com OR 2,7 (IC 95\% 1,6-4,8) - nivel de evidência 2 (Quadro 2).

A revisão sistemática de Ford ${ }^{20}$ incluiu duas revisóes sistemáticas, uma de quatro ECAC (Ford, 2008; n=392) e outra de três ECAC relativos ao OHP (Ruepert, 2011; n=326) e um ECAC (Merat, 2010; n=90). Uma das revisões incluídas ( $\mathrm{n}=392$ ) obteve um RR de 0,43 (IC 95\% 0,32-0,59), favorável ao OHP com NNT de 2-3. Na segunda revisão sistemática, um estudo ( $\mathrm{n}=101$ ) avaliou melhoria da dor abdominal (RR 2,15; IC 95\% 1,54-3,00) e dois estudos ( $\mathrm{n}=225)$ avaliaram melhoria sintomática global (RR 2,25; IC 1,7-2,98), ambos favoráveis ao OHP. Finalmente, o ECAC duplo cego $(\mathrm{n}=90)$ mostrou menor intensidade da dor/desconforto com relação ao OHP $(\mathrm{p}<0,001)$ - nível de evidência 2 (Quadro 2). 


\section{Discussão}

Em sua maioria, os estudos encontrados revelaram superioridade do OHP em relação ao placebo na melhora sintomática de indivíduos com SII. É importante salientar, no entanto, que existem ainda poucos estudos perfazendo um número reduzido de indivíduos estudados. Além disso, a evidência encontrada avalia a eficácia a curto prazo, desconhecendo-se ainda a eficácia a longo prazo ${ }^{16}$. Da mesma forma, os efeitos colaterais documentados foram transitórios e bem tolerados, no entanto não existe evidência de segurança do fármaco a longo prazo ${ }^{15,16}$.

As NOC analisadas já consideram essa terapêutica até mesmo como primeira linha de tratamento nas suas orientaçōes em relação à SII. A NOC de Jones ${ }^{17}$ não apoia a utilização desse fármaco por considerar que existe pouca evidência da sua eficácia. Deve-se salientar, no entanto, que após a publicação dessa norma foram publicados vários outros estudos favorecendo o OHP. Contudo, as sociedades científicas são cautelosas, frisando a importância de se gerar mais evidência de qualidade nessa área ${ }^{13-16}$. De fato, durante a pesquisa foram eliminadas muitas das referências por se basearem em trabalhos repetidos, verificando-se a existência de um pequeno número de ensaios clínicos utilizando o OHP. Os estudos realizados até o presente momento, embora alguns com amostras reduzidas, são de boa qualidade, duplo cegos, controlados e discutem de forma clara os seus achados e limitaçóes. Não obstante, torna-se difícil a generalizaçáo dos resultados dada a heterogeneidade metodológica e, acima de tudo, a heterogeneidade de critérios de diagnóstico para a determinação da presença de SII, bem como heterogeneidade de ferramentas utilizadas na aferição da melhoria sintomática. Os critérios de Roma III têm sido cada vez mais utilizados, tanto na clínica como em investigação, o que poderá vir a corrigir a heterogeneidade de critérios de diagnóstico de SII $^{8,9}$. Existem também questionários validados para a avaliação dos sintomas de SII ${ }^{21,22}$ que não foram usados por nenhum dos estudos, o que poderia diminuir a heterogeneidade referida pelos autores.

Todos os trabalhos analisados, à exceçâo de um $^{18}$, avaliam exclusivamente a eficácia do OHP quando comparada com placebo. A revisão de Heizer ${ }^{18}$ engloba três estudos que avaliaram a eficácia desse fármaco em comparação com a terapêutica anticolinérgica, que se revelou semelhante. Todavia, não existem até o presente momento trabalhos que comparem o OHP com outros agentes utilizados no tratamento sintomático da SII.

Apesar da dor ser condição sine qua non para o diagnóstico de SII, muitas vezes ela não é o sintoma mais debilitante para os doentes, que são mais afetados pelas alteraçôes dos hábitos intestinais ${ }^{2,14,23}$. Muitos dos estudos analisados dão muita ênfase à dor abdominal, referindo que o OHP pode ser usado tanto em indivíduos com predomínio de diarreia como de obstipação, mas não referindo se ocorre melhoria desses sintomas ${ }^{16}$. Assim, pode-se, com base nas evidências, recomendar o OHP para indivíduos cujo sintoma predominante seja a dor abdominal. Esse aspecto é também justificado pelo mecanismo de ação do OHP. O fármaco atua por antagonismo sobre os canais de cálcio do músculo liso, promovendo o seu relaxamento, e a dor abdominal da SII tem um grande componente de espasmo do músculo liso do trato gastrointestinal ${ }^{15}$.

A SII é uma patologia frequente e embora seja em geral subdiagnosticada consome um grande número de consultas em atenção primária ${ }^{4}$. Muitas das terapêuticas disponíveis para o controle sintomático têm eficácia variável, levando o doente a retornar as consultas repetidamente por persistência das queixas, pois elas afetam suas atividades diárias e sua qualidade de vida ${ }^{22,24}$. Essa falência terapêutica pode prejudicar a relação médico-paciente ${ }^{2,24}$. Assim, é crucial que seja encontrada a terapêutica que melhore a sintomatologia dos doentes e consequentemente a sua qualidade de vida. A utilização da fitoterapia, nesse caso específico, o OHP, poderá ser uma das soluçôes.

\section{Conclusão}

A evidência demonstra a eficácia do OHP na SII, especialmente se existe um componente de dor abdominal, quando comparado com o placebo (força de recomendação B). A terapêutica deve, contudo, ser adaptada a cada doente e dirigida para a sua sintomatologia predominante, uma vez que o tratamento da SII é essencialmente sintomático e comportamental ${ }^{16}$. 


\section{Referências}

1. Longstreth GF, Thompson WG, Chey WD, Houghton LA, Mearin F, Spiller RC. Functional bowel disorders. Gastroenterology. 2006; $130: 1480-1491$. http://dx.doi.org/10.1053/j.gastro.2005.11.061

2. Dumitrascu DL. Making a positive diagnosis of irritable bowel syndrome. J Clin Gastroenterol. 2011; 45: S82-85. http://dx.doi.org/10.1097/ MCG.0b013e31821fbd5a

3. Hungin AP, Whorwell PJ, Tack J, Mearin F. The prevalence, patterns and impact of irritable bowel syndrome: An international survey of 40,000 subjects. Aliment Pharmacol Ther. 2003; 17: 643-650. http://dx.doi.org/10.1046/j.1365-2036.2003.01456.x

4. Pirotta M. Irritable bowel syndrome - the role of complementary medicines in treatment. Aust Fam Physician. 2009; 38: 966-968.

5. Ford AC, Forman D, Bailey AG, Axon AT, Moayyedi P. Irritable bowel syndrome: A 10-yr natural history of symptoms and factors that influence consultation behavior. Am J Gastroenterol. 2008; 103: 1229-1239; quiz 1240. Epub 2008 Mar 1226.

6. Martin R, Barron JJ, Zacker C. Irritable bowel syndrome: Toward a cost-effective management approach. Am J Manag Care. 2001 ; 7 : S268-275.

7. Gralnek IM, Hays RD, Kilbourne A, Naliboff B, Mayer EA. The impact of irritable bowel syndrome on health-related quality of life. Gastroenterology. 2000; 119: 654-660. http://dx.doi.org/10.1053/gast.2000.16484

8. Drossman DA. The functional gastrointestinal disorders and the rome iii process. Gastroenterology. 2006; 130: 1377-1390. http://dx.doi.org/10.1053/j. gastro.2006.03.008

9. Drossman DA, Dumitrascu DL. Rome iii: New standard for functional gastrointestinal disorders. J Gastrointestin Liver Dis. 2006; 15: $237-241$.

10. Wu JC. Complementary and alternative medicine modalities for the treatment of irritable bowel syndrome: Facts or myths? Gastroenterol Hepatol. 2010; 6: 705-711.

11. INFARMED. Prontuário terapêutico online. [acesso em 2011 Nov]. Disponível em: http://www.Infarmed.Pt/prontuario/index.Php.2011.

12. Ebell MH, Siwek J, Weiss BD, Woolf SH, Susman J, Ewigman B, et al. Strength of recommendation taxonomy (sort): A patient-centered approach to grading evidence in the medical literature. Am Fam Physician. 2004; 69: 548-556.

13. Hadley SK, Gaarder SM. Treatment of irritable bowel syndrome. Am Fam Physician. 2005; 72: 2501-2506.

14. Spiller R, Aziz Q, Creed F, Emmanuel A, Houghton L, Hungin P, et al. Guidelines on the irritable bowel syndrome: Mechanisms and practical management. Gut. 2007; 56: 1770-1798. Epub 2007 May 1778. http://dx.doi.org/10.1136/gut.2007.119446

15. Kligler B, Chaudhary S. Peppermint oil. Am Fam Physician. 2007; 75: 1027-1030.

16. Quigley E, Fried M, Gwee K, Olano C, Guarner F, Khalif I, et al. Irritable bowel syndrome: A global perspective. WGO Practice Guideline; 2009

17. Jones J, Boorman J, Cann P, Forbes A, Gomborone J, Heaton K, et al. British society of gastroenterology guidelines for the management of the irritable bowel syndrome. Gut. 2000; 47: ii1-19. http://dx.doi.org/10.1136/gut.47.suppl_2.ii1

18. Heizer WD, Southern S, McGovern S. The role of diet in symptoms of irritable bowel syndrome in adults. J Am Diet Assoc. 2009; 109: $1204-1214$. http://dx.doi.org/10.1016/j.jada.2009.04.012

19. Shen YH, Nahas R. Complementary and alternative medicine for treatment of irritable bowel syndrome. Can Fam Physician. 2009; 55: 143-148.

20. Ford AC, Vandvik PO. Irritable bowel syndrome. Clin Evid. 2012; 2012: 0410.

21. Roalfe AK, Roberts LM, Wilson S. Evaluation of the birmingham ibs symptom questionnaire. BMC Gastroenterol. 2008; 8: 30. http://dx.doi. org/10.1186/1471-230X-8-30

22. Camilleri M. Drug development and ibs drugs: Experience from the past, current challenges, and proposal for the future. Curr Opin Pharmacol. 2008; 8: 671-676. Epub 2008 Jul 2009. http://dx.doi.org/10.1016/j.coph.2008.06.001

23. Malone MA. Irritable bowel syndrome. Prim Care. 2011; 38: 433-447; viii. http://dx.doi.org/10.1016/j.pop.2011.05.003

24. Olden KW. Approach to the patient with severe, refractory irritable bowel syndrome. Curr Treat Options Gastroenterol. 2003; 6: 311-317. http:// dx.doi.org/10.1007/s11938-003-0023-8 